Cite this: Analyst, 2013, 138, 5288

Received 14th June 2013

Accepted 10th July 2013

DOI: 10.1039/c3an01179h

www.rsc.org/analyst

\section{Solid-state Forensic Finger sensor for integrated sampling and detection of gunshot residue and explosives: towards 'Lab-on-a-finger'}

\author{
Amay J. Bandodkar, Aoife M. O'Mahony, Julian Ramírez, Izabela A. Samek, \\ Sean M. Anderson, Joshua R. Windmiller and Joseph Wang*
}

Increasing security needs require field-deployable, on-the-spot detection tools for the rapid and reliable identification of gunshot residue (GSR) and nitroaromatic explosive compounds. This manuscript presents a simple, all-solid-state, wearable fingertip sensor for the rapid on-site voltammetric screening of GSR and explosive surface residues. To fabricate the new Forensic Fingers, we screen-print a threeelectrode setup onto a nitrile finger cot, and coat another finger cot with an ionogel electrolyte layer. The new integrated sampling/detection methodology relies on 'voltammetry of microparticles' (VMP) and involves an initial mechanical transfer of trace amounts of surface-confined analytes directly onto the fingertip-based electrode contingent. Voltammetric measurements of the sample residues are carried out upon bringing the working electrode (printed on the index finger cot) in direct contact with a second finger cot coated with an ionogel electrolyte (worn on the thumb), thus completing the solidstate electrochemical cell. Sampling and screening are performed in less than four minutes and generate distinct voltammetric fingerprints which are specific to both GSR and explosives. The use of the solid, flexible ionogel electrolyte eliminates any liquid handling which can resolve problems associated with leakage, portability and contamination. A detailed study reveals that the fingertip detection system can rapidly identify residues of GSR and nitroaromatic compounds with high specificity, without compromising its attractive behavior even after undergoing repeated mechanical stress. This new integrated sampling/detection fingertip strategy holds considerable promise as a rapid, effective and low-cost approach for on-site crime scene investigations in various forensic scenarios.

\section{Introduction}

Decentralized, easy-to-use and rapid tools for forensic analysis have become of utmost importance in view of the recent increase in international and domestic crimes related to firearms and explosives. ${ }^{\mathbf{1 - 4}}$ Traditionally, such analysis has been done in a central laboratory, which mandates time-consuming sampling, transportation and storage steps. ${ }^{\mathbf{1 , 5}}$ These limitations result in sparse deployment of equipment and can cause delays in crime scene investigations. Furthermore, transportation and storage steps may cause contamination/degradation of the collected samples, thus jeopardizing the forensic investigation and the effective administration of justice. ${ }^{1}$

Currently, analytical methods such as X-ray Fluorescence $(\mathrm{XRF})^{6,7}$ and Raman spectroscopy ${ }^{8}$ have come closest to the realization of portable devices for forensic analysis. However, these devices are still complex, cumbersome and costly. Laser Induced Breakdown Spectroscopy (LIBS) is also an attractive

Department of NanoEngineering, University of California, San Diego, La Jolla, CA 92093, USA. E-mail: josephwang@ucsd.edu; Fax: +1-858-534-9553; Tel: +1-858-2460128 technique for developing a field-deployable sensing system, but such a device is yet to be demonstrated., ${ }^{\mathbf{9}, 10}$ On the other hand, electroanalytical devices require simple instrumentation that can be consolidated into a small footprint due to advances in microelectronics, while generating reproducible and specific signals towards electroactive analytes. Electroanalysis can thus be exploited to develop portable analytical tools using sensitive and inexpensive sensors that offer attractive opportunities for diverse decentralized forensic applications, ranging from 'alternative-site' testing (e.g., at a crime scene) to police-station screening. ${ }^{\mathbf{1} 4}$ Recently, we demonstrated a protocol based on voltammetry of microparticles (VMP) (formerly known as abrasive voltammetry) for detecting GSR with screen printed sensors. ${ }^{11}$ However, this technique does not involve a wearable device, and still mandates the use of aqueous media and related liquid handling for detection, making the process somewhat cumbersome. An all-solid-state wearable sensor could provide a suitable, robust solution to this limitation.

Two major challenges confound the development of an allsolid-state wearable forensic sensor: (i) the first seventy-two hours are the most critical period in forensic investigations as potential evidence not collected initially is often lost; ${ }^{7}$ rapid 

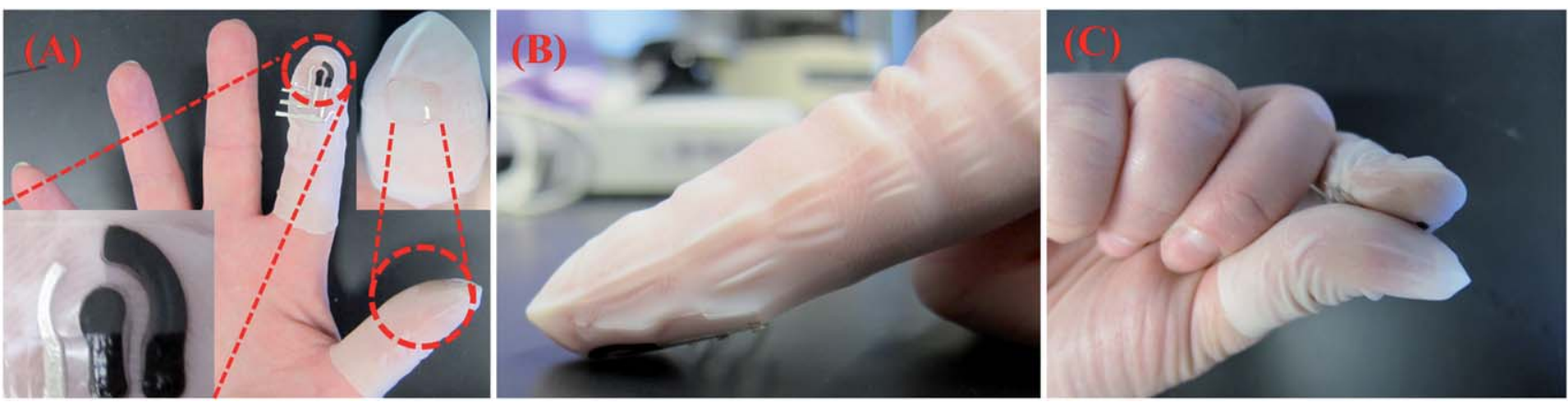

Fig. 1 Schematic delineating voltammetry of microparticles at a wearable Forensic Finger. (A) The Forensic Finger exhibiting the three electrode surface screen-printed onto a flexible nitrile finger cot (bottom left inset), as well as a solid, conductive ionogel immobilized upon a similar substrate (top right inset); (B) 'swipe' method of sampling to collect the target powder directly onto the electrode; (C) completion of the electrochemical cell by joining the index finger with electrodes to the thumb coated with the solid ionogel electrolyte.

sample collection is thus a crucial step. ${ }^{2,11,12}$ (ii) Many electrochemical techniques mandate the use of a solvent electrolyte. This is a major inconvenience as liquid handling systems can suffer from problems associated with leakage and contamination. Hence, a solid-state electrolyte, incorporated onto the wearable substrate, can circumvent the need for carrying additional aqueous reagents, and facilitate rapid decentralized forensic investigations.

This article describes an innovative approach that leverages fingertip sensors for rapid on-site detection of explosives and gunshot residue (GSR). The new single-use wearable fingertip sensor, called a Forensic Finger, includes an entire electrochemical cell (electrode contingent + solid-state electrolyte) fabricated on stretchable and flexible finger cots (Fig. 1A). The electrode contingent is fabricated on the tip of a finger cot using well-established screen printing technology. The new concept builds upon our expertise in printed, wearable sensors ${ }^{13}$ and electroanalysis of forensic samples. ${ }^{\mathbf{1} 11}$ Wearable electronic devices have received tremendous attention over the last decade. Researchers have successfully developed electronic textiles for monitoring vital physiological parameters, such as heart rate, body temperature, ECG and patient movement, ${ }^{14,15}$ sweat rate ${ }^{16}$ and sweat electrolytes. ${ }^{17,18}$ Epidermal sensors have also recently gained importance with groups demonstrating tattoo-based devices for monitoring physiologically-relevant physical $^{19}$ and chemical $^{20}$ parameters. However, a wearable chemical forensic device for detecting explosives and GSR is yet to be demonstrated.

For simple and reliable sampling the new printable Forensic Finger utilizes the VMP method. VMP involves mechanical transfer of materials directly onto the surface of a solid electrode followed by voltammetric measurements of the collected sample. ${ }^{21}$ The advantages associated with this sampling method include convenience of qualitative determination of sparingly soluble and insoluble species, small sample requirements and application to a variety of solid compounds for electroanalysis. ${ }^{22-24}$ To obviate the need for an aqueous electrolyte, we synthesize a solid-state ionogel, which is cast directly onto the tip of a finger cot. Ionogels are defined as solid or gel-like inorganic materials that endow room temperature ionic liquids
(RTILs) with structure and dimensional capability. ${ }^{25-28}$ These represent attractive electrolyte materials owing to their low cost, mechanical flexibility and ionic conductivity.

Following the fabrication of the Forensic Finger, the user adorns the electrode-printed finger cot on the index finger and the ionogel-modified finger cot on the thumb (Fig. 1A). To investigate a surface for possible GSR/explosives residues, the user gently abrades the index finger - containing the finger cot with the printed electrodes - on the surface (Fig. 1B) and then brings it in contact with the ionogel-coated thumb to complete the electrochemical cell (Fig. 1C). The sample is then analyzed using rapid square wave voltammetry with a field-portable electrochemical analyzer. The complete process can be carried out independently by the user within four minutes.

This printable fingertip sensor was characterized for the detection of GSR (from live bullet casings) and 2,4-dinitrotoluene (DNT) powder residues. Detailed studies demonstrate the high specificity of the Forensic Finger sensor towards the target analytes, the stability of the ionogel, and the robustness of the system towards mechanical stress. Finally, the Forensic Finger was employed for on-site detection of GSR at a local firing range. The new forensic tool thus aims to support on-site criminal investigations, by rapidly identifying suspected explosive or gunshot residues (without a detailed quantitation). Its attractive analytical performance, illustrated in the following sections, during laboratory and in-field testing, along with its ergonomics, low-cost, and robustness, opens up new avenues in wearable devices for decentralized crime scene investigation.

\section{Materials and methods}

\subsection{Reagents and materials}

1-Ethyl-3-methylimidazolium tetrafluoroborate $\left(\mathrm{C}_{2} \mathrm{mimBF}_{4}\right)$, 2,4-dinitrotoluene (DNT) and poly(ethylene glycol) diacrylate (PEGDA, Mw $=700)$ were purchased from Sigma-Aldrich (St. Louis, MO). Irgacure 2959 (initiator) was purchased from BASF Vandalia (Greenville, OH). Nitrile Finger Cots $(4404 \mathrm{~L})$ were obtained from Tech Med Services Inc. (Hauppauge, NY). Remington UMC Target 45 automatic ammunition (containing 16$82 \%$ copper, $14-79 \%$ lead, and $0.1-2 \%$ antimony) was 
purchased from P2K shooting range (El Cajon, CA), and was used to obtain GSR samples (deposited in the shooting range) for laboratory-based experiments. Conductive $\mathrm{Ag} / \mathrm{AgCl}$ (E2414), carbon (E3449), and insulator inks (E6165) were procured from Ercon Inc. (Wareham, MA) and a transparent dielectric ink (5036) was obtained from DuPont Inc. (Wilmington, DE).

All laboratory-based electrochemical measurements were performed using a $\mathrm{CH}$ Instruments (Austin, TX) model 630C electrochemical analyzer while a portable $\mathrm{CH}$ Instruments model 1230A electrochemical analyzer was used for field-based GSR assessment.

\subsection{Fabrication and detection protocol for Forensic Finger}

The sensors were fabricated using an MPM SPM semi-automatic screen printer (Speedline Technologies, Franklin, MA). The sensor patterns were designed in AutoCAD (Autodesk, San Rafael, CA) and outsourced for fabrication on $75 \mu \mathrm{m}$-thick stainless steel stencils (Metal Etch Services, San Marcos, CA). A separate stencil pattern was created for each layer $(\mathrm{Ag} / \mathrm{AgCl}$, carbon, insulator). Prior to screen printing the sensors, the finger cots were stretched over an alumina substrate to provide a flat surface for high resolution printed electrodes. They were secured using polyester tape. The first step in the sensor fabrication process involved the printing of a layer of transparent dielectric ink. This layer was printed to impart further mechanical resiliency to the underlying finger cot and the electrode system. This was followed by printing layers of $\mathrm{Ag} /$ $\mathrm{AgCl}$, carbon, and finally a transparent dielectric ink to define the active electrode area. Following each routine, the ink was cured at $90{ }^{\circ} \mathrm{C}$ for $10 \mathrm{~min}$. The contacts for the sensor interface to the electrochemical analyzer were obtained by screen printing $\mathrm{Ag} / \mathrm{AgCl}$ on a flexible PET substrate, which was subsequently cured at $90{ }^{\circ} \mathrm{C}$ for $10 \mathrm{~min}$. The $\mathrm{Ag} / \mathrm{AgCl}$-coated PET substrate was later cut into rectangular strips and fixed to the printed sensor on the finger cots using conductive silver adhesive (MG Chemicals, Ontario, Canada). The conductive adhesive was cured at $65{ }^{\circ} \mathrm{C}$ for $10 \mathrm{~min}$.

The ionogel was synthesized according to a reported method. ${ }^{29}$ In brief, $20 \mathrm{wt} \%$ PEDGA and $2 \mathrm{wt} \%$ initiator were mixed with $\mathrm{C}_{2} \mathrm{mimBF}_{4}$. The composition was sonicated for $30 \mathrm{~min}$ to obtain a uniform mixture. Later, $10 \mu \mathrm{L}$ of the mixture was spread over $1 \times 1 \mathrm{~cm}^{2}$ area of the finger cot (stretched and attached to an alumina substrate, similar to the screen printing step) and irradiated with a UV light using a PortaRay 400R curing system (Uvitron International, Inc., West Springfield, MA) for $30 \mathrm{~s}$, leading to photo-crosslinking of the PEGDA to obtain the ionogel. Fig. 1A shows the actual photo of both the finger sensor and the ionogel.

The finger cot containing the printed sensor was worn on the index finger and the ionogel-coated finger cot was worn on the thumb of the same hand for the detection of GSR or explosives (Fig. 1A). The index finger (with printed sensor) was abrasively rubbed over a surface (possibly containing the analyte powder) in a manner which allowed some of the powder to be mechanically transferred onto the active area of the sensor (Fig. 1B). For GSR analysis the Forensic Finger was abrasively rubbed over Remington UMC Target 45 automatic ammunition, on which GSR was present. In the case of explosives detection, the sensor was abraded in a similar fashion over a DNT powder (as received form the manufacturer). Later, the thumb (with ionogel) was brought in contact with the index finger (Fig. 1C) to achieve a complete electrical circuit. Each Forensic Finger was used for one-time analysis. Separate sensors were used for the detection of DNT and GSR.

\subsection{Square-wave anodic stripping voltammetry}

Square Wave Stripping Voltammetry (SWSV) was employed to characterize the electrochemical signature of GSR. A potential of $-0.95 \mathrm{~V}$ was applied for $120 \mathrm{~s}$, and a scan to a final potential of $0 \mathrm{~V} v s$. $\mathrm{Ag} / \mathrm{AgCl}$ was performed. An accumulation time was implemented for deposition of metal ions present in GSR alongside metallic species. Square-Wave Voltammetry (SWV) was employed to characterize the electrochemical signature of DNT. The voltammograms were scanned from an initial potential of $0 \mathrm{~V}$ to a final potential of $-1.75 \mathrm{~V} v s$. $\mathrm{Ag} / \mathrm{AgCl}$. All scans were performed at a frequency of $25 \mathrm{~Hz}$, an amplitude of $25 \mathrm{mV}$, a potential step of $4 \mathrm{mV}$ and using the ionogel described in Section 2.2.

\section{Results and discussion}

In this work, GSR and DNT were electrochemically analyzed at a bare carbon electrode, screen-printed onto a disposable finger cot substrate. Fig. 1 outlines the sequence for sampling and analysis at these new Forensic Finger sensors. In Fig. 1A, we observe the three electrode cell printed onto the disposable finger cot worn on the index finger. The working and counter electrodes are comprised of carbon ink while the reference electrode is comprised of $\mathrm{Ag} / \mathrm{AgCl}$ ink. The fabrication of this three-electrode cell is discussed in Section 2.2. We also observe the ionogel electrolyte, which is immobilized upon a finger cot worn on the thumb. Fig. 2B illustrates the abrasive sampling method which involves 'swiping' the index-finger electrode over the surface of interest, transferring the target sample directly upon the working electrode, which is then immediately ready for analysis. This 'swiping' protocol coupled with analysis is described in Section 1 and labeled voltammetry of microparticles (VMP). By placing the ionogel-electrolyte thumb in direct contact with the three-electrode index finger, the
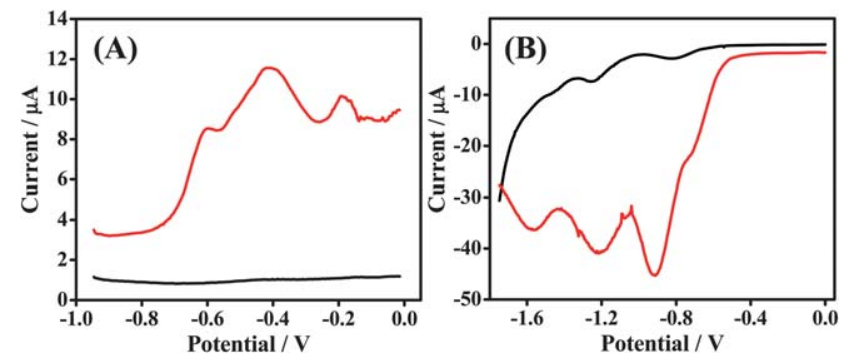

Fig. 2 Voltammetric response obtained at Forensic Finger sensor/ionogel interface in the absence (black) and in the presence (red) of (A) GSR \& (B) DNT. Voltammetric parameters are outlined in Section 2.3 
electrochemical cell is completed and is ready for immediate analysis, as illustrated in Fig. 1C.

Utilizing SWSV, as outlined in Section 2.3, we observe a distinct pattern corresponding to traces of GSR from the surface of ammunition (described in Section 2.1). Fig. 2A outlines a voltammogram for the ionogel in the absence of GSR (black) as well as the voltammetric fingerprint recorded subsequently to swiping a GSR-rich surface (red). The voltammetric pattern for this GSR sweep is very distinct and has been shown to be characteristic from scan to scan. We observe three voltammetric signals at potentials $-0.6 \mathrm{~V},-0.4 \mathrm{~V}$ and $-0.2 \mathrm{~V}$, which are attributed to $\mathrm{Pb}, \mathrm{Sb}$, and $\mathrm{Cu}$, respectively. Previous data demonstrates that these metals strip at similar potentials on a similar electrode surface. ${ }^{11}$ We also attain the voltammetric signature of DNT by scanning reductively upon swiping the Forensic Finger over a DNT-rich surface. Fig. 2B displays voltammograms for the reductive scan of the ionogel in the absence (black) and in the presence (red) of DNT. The electrochemical fingerprint of this nitroaromatic compound is very distinct compared with that of the blank ionogel scan due to the presence of easily-reducible nitro groups. Three signals are observed for this reductive scan at potentials $-0.9 \mathrm{~V},-1.2 \mathrm{~V}$ and $-1.6 \mathrm{~V}$. The first two signals at $-0.9 \mathrm{~V}$ and $-1.2 \mathrm{~V}$ are attributed to the stepwise reduction of the two nitro groups of DNT to hydroxylamine groups, while the third signal (at $-1.6 \mathrm{~V}$ ) is attributed to the reduction of one of the hydroxylamine groups to an amine..$^{30,31}$

Employing this system, several studies were conducted to examine the specificity of the Forensic Finger sensor toward the target analytes, the stability of the ionogel over several days, and the robustness of the system towards mechanical stress. Finally, real GSR samples were examined from different surfaces at a firing range to demonstrate the field-deployable nature of this system.

\subsection{Specificity of Forensic Finger toward GSR and DNT}

We examined the specificity of the Forensic Finger toward both GSR and DNT compared with VMP of other surfaces to ensure that the voltammetric signals are due to the target components and not other contaminants that may be routinely encountered. These control surfaces were obtained from random objects in the laboratory, and no attempt was made to clean or alter them. In Fig. 3(A-E), we examine the specificity of the system for the anodic scan comparing a GSR-rich surface with other surfaces upon which potential contaminants could be immobilized. Fig. 3C shows SWSV for the oxidation of species found in GSR samples. Three voltammetric signals are observed upon swiping the GSR-rich surface. The signal at $-0.6 \mathrm{~V}$ is attributed to $\mathrm{Pb}$, the signal at $-0.4 \mathrm{~V}$ is attributed to $\mathrm{Sb}$, and the signal at $-0.2 \mathrm{~V}$ is attributed to $\mathrm{Cu}$, as observed in Fig. 2A. Fig. 3A, B, D and E display VMP signals of wooden, plastic, paper and metal surfaces, respectively. A featureless baseline is observed for samples from each of these control surfaces, clearly indicating the absence of false response and substantiating that the voltammetric fingerprint observed in Fig. 3C is due to the presence of GSR. These specificity tests outline the suitability of this protocol for the detection of GSR in forensic scenarios.

Similarly, in Fig. 3( $\left.\mathrm{A}^{\prime}-\mathrm{E}^{\prime}\right)$, we demonstrate the specificity of the Forensic Finger in the identification of DNT powder. For example, Fig. 3C' displays reductive SWV recorded following swiping of a DNT powder sample. The distinctive reduction signals of DNT are observed at potentials $-0.9 \mathrm{~V},-1.2 \mathrm{~V}$ and $-1.6 \mathrm{~V}$, corresponding to the stepwise reduction of the nitrogroups to hydroxylamine groups and further reduction to an amine, as previously discussed in Section 3. This voltammetry can be compared to the featureless responses obtained for samples of wooden, plastic, paper and metal surfaces,

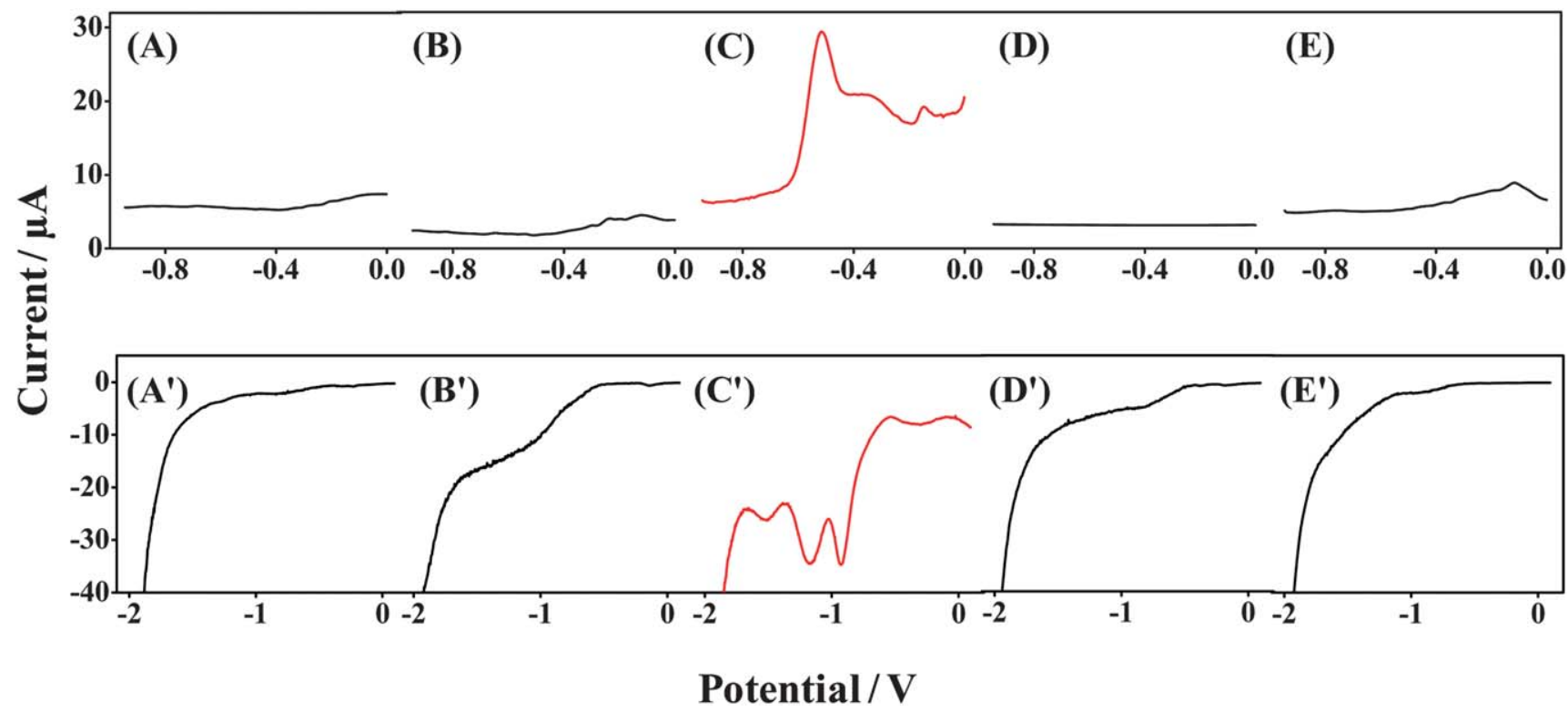

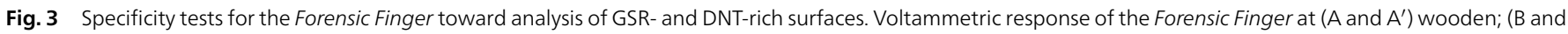
$B^{\prime}$ ) plastic; (C) GSR-rich; ( $\left.C^{\prime}\right)$ DNT-rich; (D and $D^{\prime}$ ) paper and ( $E$ and $E^{\prime}$ ) metal surfaces. 
provided in Fig. $3 \mathrm{~A}^{\prime}, \mathrm{B}^{\prime}, \mathrm{D}^{\prime}$ and $\mathrm{E}^{\prime}$, respectively, which again indicates the absence of false signals. Based on the data represented in Fig. 3, it is clear that the Forensic Finger is well suited for field-based analysis of various security-related compounds.

\subsection{Examination of stability of ionogel for detection of GSR and DNT}

The advantages of utilizing ionogels as electrolytes for wearable electrodes are outlined in Section 1 . One of the most significant advantages of the use of an ionogel electrolyte is the thermal and kinetic stability of this media due to the negligible vapor pressure of the ionic liquid..$^{32}$ In order to fully evaluate this with respect to the Forensic Finger, we examined the ionogel's performance on the day of fabrication (Day 1), and 6 days thereafter (Day 7), to evaluate stability and shelf-life. The results of this study are outlined in Fig. 4. Fig. 4A \& B display the results for examination of the GSR electrochemical signature corresponding to Day 1 and Day 7 after fabrication, respectively. A featureless baseline is obtained at both time intervals for the ionogel in the absence of the GSR sample (black line). This substantiates the ionogel's stability over this period. We also observe a clear voltammetric fingerprint for GSR sampling (red line) for Days 1 and 7. The three voltammetric signals corresponding to $\mathrm{Pb}, \mathrm{Sb}$ and $\mathrm{Cu}$, observed in previous sections, are clear for each period, albeit the levels of the species present vary from scan to scan. This reflects the limited control over the levels of sample that are transferred using the swipe sampling technique. However, this protocol is offered as a rapid field screening tool, leading to a distinct voltammetric signature for suspected powders whereby a threshold level is set for the presence or absence of the target analyte, and no attempt at quantification is made.

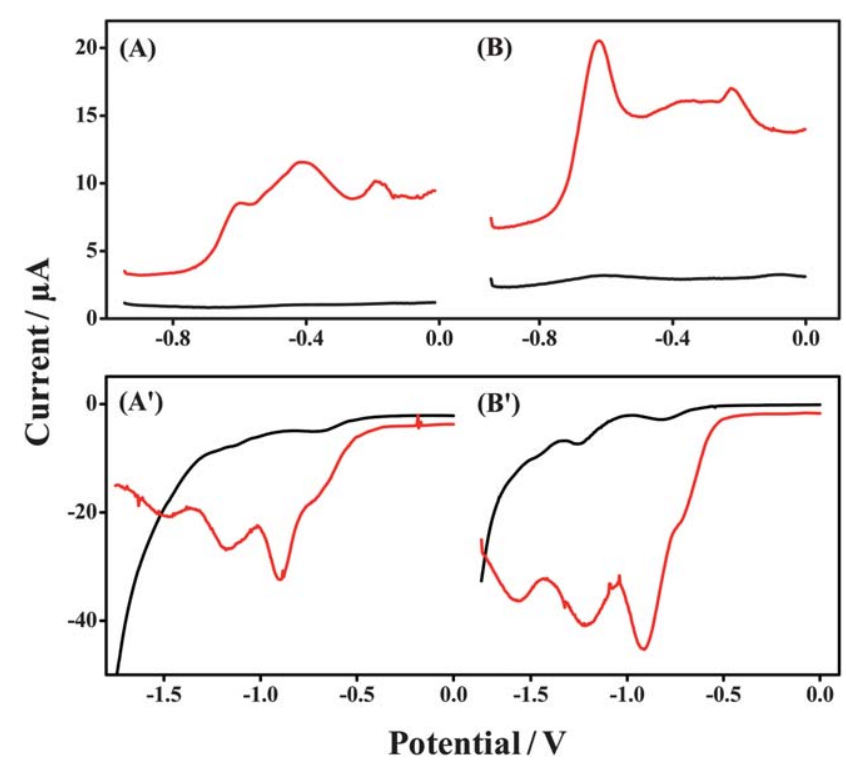

Fig. 4 Week-long stability study of the ionogel. Response in Days 1 ( $A$ and $A^{\prime}$ ) and 7 ( $B$ and $\left.B^{\prime}\right)$, voltammetry of bare sensor (black line), GSR sampling (red line $A \& B$ ), and DNT sampling (red line $-A^{\prime} \& B^{\prime}$ ).
Fig. $4 \mathrm{~A}^{\prime}$ and $\mathrm{B}^{\prime}$ display the results for examination of the DNT signature for Day 1 and Day 7, respectively. As per the GSR test, a featureless baseline response of the ionogel alone (black line) is recorded for each of the time intervals showing no degradation of the electrolyte within the gel matrix. In addition, clear reduction signals are observed for DNT (red line) for each time interval, illustrating the appreciable thermal and kinetic stability of the ionogel. The three characteristic reduction signals for DNT, observed in previous sections, remain clear and well-defined (although the actual peak heights change, as expected from changes in the amount of collected DNT). These observations highlight the significant advantage of implementing ionogels into a field-deployable tool, as negligible care is required for the storage of this electrolyte. While this study was not extended beyond seven days, it is still expected that the ionogel would retain its stability given the thermal and kinetic properties as well as negligible vapor pressure of RTILs in general. ${ }^{32}$

\subsection{Stress study to examine strength of Forensic Finger}

The Forensic Finger is presented as a wearable sensor suitable for field-deployable analysis. Therefore, the sensor itself must be resilient against the movements of the wearer. In this study, we examined the effects of mechanical stress upon the response of the electrode system for the detection of both GSR and DNT.

The stress applied to the electrode involved iterations of opening and closing a fist, while wearing the finger cot electrode, as shown in Fig. 5A (open) and B (closed). Both GSR and DNT were sampled after applying mechanical stress iterations to the sensor. A finger cot electrode was subject to 10 flexions of the wearer's hand, after which GSR was sampled. A voltammetric scan was then taken of the GSR sample and the result is

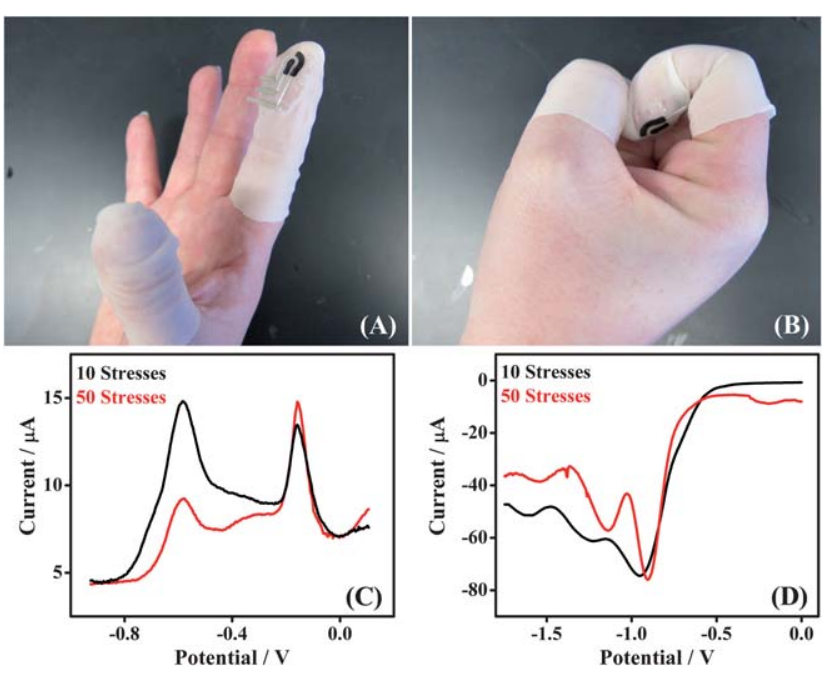

Fig. 5 Effect of mechanical stress applied to the Forensic Finger. (A) Opening and (B) closing of a fist, while wearing the finger cot electrode. (C) SWSV scans of GSR sampled from a GSR-rich surface subsequent to 10 (black) and 50 (red) applications of mechanical stress to the electrode. (D) SWV scans of a DNT sample subsequent to 10 (black) and 50 (red) applications of mechanical stress. 

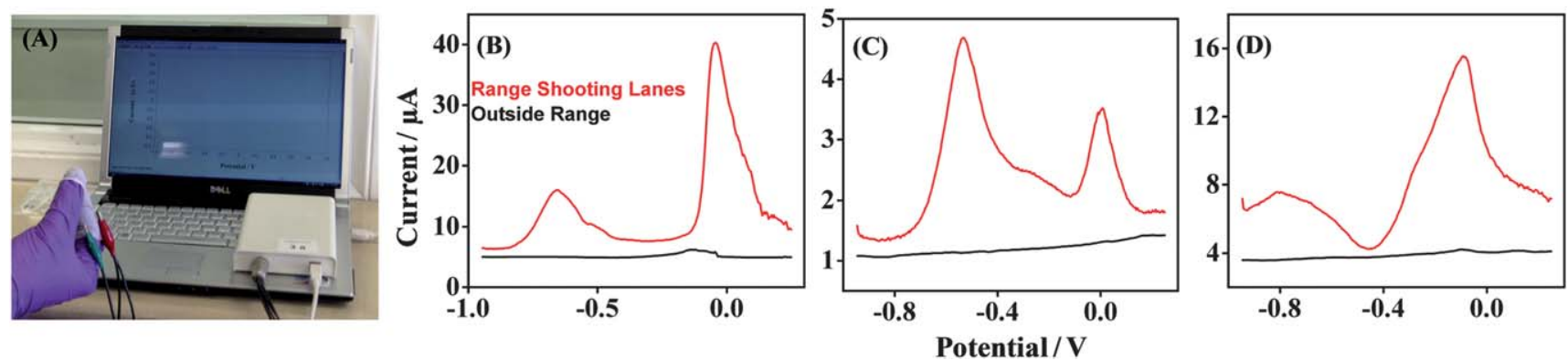

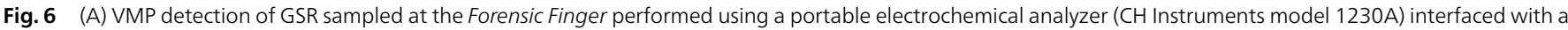

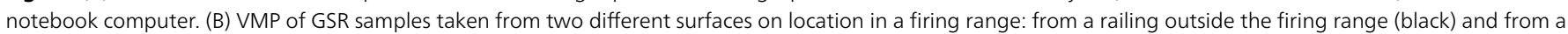

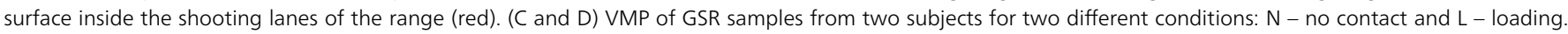
Electrochemical parameters, as in Section 2.3. See Experimental section for other details.

shown in Fig. 5C (black). A second finger cot electrode was subject to 50 flexions of the wearer's hand, after which GSR was again sampled. Subsequent voltammetry was implemented on the sample and the response is also displayed in Fig. 5C (red). From an inspection of these scans, one can notice that the distinct electrochemical signature for GSR does not appear to be affected by the repeated mechanical stress upon either of the electrodes, regardless of flexion number. There is negligible change of the GSR signature with increasing mechanical stress iterations (10-black and 50-red), underscoring the robust nature of the electrode. A similar experiment was performed utilizing DNT as the target analyte, and the results are provided in Fig. 5D. As with the GSR, negligible degradation of the DNT profile was observed with increasing mechanical stress for varying iterations (10-black and 50-red). The voltammetric fingerprint for each species is consistent with each stress evaluation, substantiating the stability and practicality of this fingertip electrode as a wearable sensor in a variety of practical scenarios.

\subsection{In-field analysis of Forensic Finger toward detection of gunshot residue}

A study was executed at a shooting range to examine the application of the Forensic Finger in a real-life scenario involving GSR detection. The effect of the VMP technique for the detection of GSR over different surfaces was examined, as well as variations in voltammetric GSR fingerprints from subjects who handled a firearm. All the studies were performed using a portable electrochemical analyzer interfaced with a notebook computer, as displayed in Fig. 6A. The first surface was sampled outside the shooting range, where trace GSR may be found, but where no firearms were being discharged. The voltammetry obtained for this sample with the fingertip sensor is shown in Fig. 6B (black) and displays a relatively featureless baseline within the scope of the experiment. A trace $\mathrm{Cu}$ signal, observed at $-0.15 \mathrm{~V}$, may be due to the transport of GSR particles outside of the range facility. The second sample was taken over a wooden surface within the shooting lanes of the range, and is also shown in Fig. 6B (red). This scan displays a significant increase in the levels of GSR, as expected from a GSR-rich environment. Two voltammetric signals are observed at potentials $-0.6 \mathrm{~V}$ and $0 \mathrm{~V}$, and are attributed to $\mathrm{Pb}$ and $\mathrm{Cu}$, respectively. A clear signal is not observed for $\mathrm{Sb}$, however, contributions from $\mathrm{Sb}$ are indicated by the shift of the Cu peak potential to a more positive value. ${ }^{11}$ This voltammetry demonstrates the potential use of the Forensic Finger in a scenario whereby a more GSR-rich environment at a crime scene can be identified, signifying the discharge of a firearm in one particular location. The ability of the VMP technique to detect GSR from the hand of an individual, before and after handling a firearm, has also been investigated. Samples were obtained from a subject at different instances during the experimental process: in the laboratory, prior to any contact with GSR, named $\mathrm{N}$ - no contact, and having handled and loaded the firearm, named L - loading. These control scenarios were sampled from two different subjects, and the results are shown in Fig. 6C and D. Fig. 6C illustrates the control samples taken from one subject's right hand, and Fig. 6D displays the samples taken from a second subject's right hand. The $\mathrm{N}$ - no contact voltammetry (black) displays a featureless baseline within the scope of the experiment, indicating the absence of any GSR-relevant components on the subject's hands. The $\mathrm{L}$ - loading voltammetric response (red) displays two distinct signals at $-0.6 \mathrm{~V}$ and $0 \mathrm{~V}$ which are attributed to $\mathrm{Pb}$ and $\mathrm{Cu}$ (but with contributions from $\mathrm{Sb}$ as previously outlined), respectively. The $\mathrm{Pb}$ and $\mathrm{Cu}$ signals are similar and consistent with those observed in Fig. 6B and as before, display a significant fingerprint for GSR when compared to the 'no contact' scenario. The increase in these signals is consistent over two different subject samples and demonstrates that GSR can easily be transported from the surface of a firearm and ammunition to a subject merely by handling and loading. Similar voltammetric fingerprints for these control scenarios have been reported at screen-printed electrodes utilizing a liquid aqueous electrolyte. ${ }^{11}$ Variations in the current output of the signatures also demonstrate that sampling can vary, and different amounts of GSR can be obtained at different times. However, the aim of this study is to demonstrate a rapid screening tool, and it is clear that the variation in voltammetry prior to and following handling a firearm is dramatically different. The Forensic 
Finger concept thus represents a promising new avenue towards the on-site detection of GSR.

\section{Conclusions}

We have demonstrated the fabrication and characterization of a wearable fingertip sensor - Forensic Finger - for field-deployable, on-the-spot analysis of GSR and explosive agents. The new concept offers a convenient, integrated sampling and analysis routine, which can be performed within minutes, and obviates the necessity for liquid handling to realize a complete, userfriendly device. The Forensic Finger consists of electrodes, screen printed upon a stretchable finger cot substrate and is complimented with a conductive, flexible ionogel electrolyte. Sampling is implemented by utilizing the simple and efficient VMP method, whereby target analytes are mechanically transferred directly onto the electrode surface via swiping the sensor over the area of interest. The Forensic Finger exhibits noteworthy sensitivity and selectivity toward both GSR and DNT. Studies have demonstrated that the ionogel electrolyte is stable over a week-long period. We demonstrate the robustness of the threeelectrode Forensic Finger sensor through mechanical stress studies and illustrate that the characteristic voltammetry of both GSR and DNT is retained. The integrated sampling and analysis steps, along with the removal of liquid handling and rapid square-wave voltammetry, ensure results within a four minute time frame. We have demonstrated the practical application of this fingertip sampling/detection system by presenting the distinct voltammetric response for GSR in a 'GSR-rich' environment as well as the voltammetric fingerprint of GSR immobilized from the hand of a subject subsequent to the handling of a firearm. The new concept holds considerable promise as a portable, field-deployable screening method aimed at the rapid identification of a security threat or providing forensic evidence from either firearms or explosives (without detailed quantitative information). We anticipate future integration of the controlled electronic backbone in the form of a wristband, wristwatch, or ring. With the rapid development of wireless communications, the transmission of results to a smartphone or centralized database would be of substantial utility to on-site forensic investigations.

\section{Acknowledgements}

This material is based upon work supported under Contract no. HQ0034-11-C-0034. Any opinions, findings and conclusions or recommendations expressed in this material are those of the author(s) and do not necessary reflect the views of the Washington Headquarters Services-Acquisition Directorate [Assistant Secretary of Defense for Research and Engineering (Defense Biometrics and Forensics Office)]. J. R. and J. R. W. acknowledge support from the NIGMS IMSD program and William J. von Liebig Center under the DOE-sponsored Southern California Clean Energy Technology Acceleration Program, respectively. Special thanks are due to Prof. Shaochen Chen (UCSD) for providing PEDGA and initiator. We would also like to thank the staff at the $\mathrm{P} 2 \mathrm{~K}$ gun range (El Cajon, CA) for their cooperation.

\section{References}

1 A. M. O'Mahony and J. Wang, Electroanalysis, 2013, 25, 13411358.

2 H.-H. Meng and B. Caddy, J. Forensic Sci., 1997, 42, 553-570.

3 A. M. O'Mahony and J. Wang, Anal. Methods, 2013, DOI: 10.1039/c3ay40636a, Advance Article.

4 J. Wang, Electroanalysis, 2007, 19, 415-423.

5 J. Wang, B. Tian, J. Wang, J. Lu, C. Olsen, C. Yarnitzky, K. Olsen, D. Hammerstrom and W. Bennett, Anal. Chim. Acta, 1999, 385, 429-435.

6 K. Nakano, C. Nishi, K. Otsuki, Y. Nishiwaki and K. Tsuji, Anal. Chem., 2011, 83, 3477-3483.

7 J. Schweitzer, J. I. Trombka, S. Floyd, C. Selavka, G. Zeosky, N. Gahn, T. McClanahan and T. Burbine, Nucl. Instrum. Methods Phys. Res., Sect. B, 2005, 241, 816-819.

8 J. Bueno, V. Sikirzhytski and I. K. Lednev, Anal. Chem., 2012, 84, 4334-4339.

9 J. R. Almirall, S. Umpierrez, W. Castro, I. Gornushkin and J. Winefordner, Sensors and C3I Technologies for Homeland Security and Homeland Defense IV, Proc. SPIE, 2005, 5778, 657-666.

10 C. R. Dockery and S. R. Goode, Appl. Opt., 2003, 42, 61536158.

11 A. M. O'Mahony, J. R. Windmiller, I. A. Samek, A. J. Bandodkar and J. Wang, Electrochem. Commun., 2012, 23, 52-55.

12 O. Dalby, D. Butler and J. W. Birkett, J. Forensic Sci., 2010, 55, 924-943.

13 J. R. Windmiller and J. Wang, Electroanalysis, 2013, 25, 2946.

14 G. López, V. Custodio and J. I. Moreno, IEEE Trans. Inf. Technol. Biomed., 2010, 14, 1446-1458.

15 D. Curone, E. L. Secco, A. Tognetti, G. Loriga, G. Dudnik, M. Risatti, R. Whyte, G. Magenes and A. Bonfiglio, IEEE Trans. Inf. Technol. Biomed., 2010, 14, 694-701.

16 P. Salvo, F. Di Francesco, D. Costanzo, C. Ferrari, M. G. Trivella and D. De Rossi, IEEE Sens. J., 2010, 10, 1557-1558.

17 B. Schazmann, D. Morris, C. Slater, S. Beirne, C. Fay, R. Reuveny, N. Moyna and D. Diamond, Anal. Methods, 2010, 2, 342-348.

18 V. F. Curto, S. Coyle, R. Byrne, N. Angelov, D. Diamond and F. Benito-Lopez, Sens. Actuators, B, 2012, 171-172, 13271334.

19 D. H. Kim, N. Lu, R. Ma, Y. S. Kim, R. H. Kim, S. Wang, J. Wu, S. M. Won, H. Tao, A. Islam, K. J. Yu, T. Kim, R. Chowdhury, M. Ying, L. Xu, M. Li, H. J. Chung, H. Keum, M. McCormick, P. Liu, Y. W. Zhang, F. G. Omenetto, Y. Huang, T. Coleman and J. A. Rogers, Science, 2011, 333, 838-843.

20 A. J. Bandodkar, V. W. S. Hung, W. Jia, G. Valdés-Ramírez, J. R. Windmiller, A. G. Martinez, J. Ramírez, G. Chan, K. Kerman and J. Wang, Analyst, 2013, 138, 123-128.

21 T. Grygar, F. Marken, U. Schroder and F. Scholtz, Collect. Czech. Chem. Commun., 2002, 67, 163-208.

22 F. Scholz, L. Nitschke and G. Henrion, Naturwissenschaften, 1989, 76, 167-168. 
23 F. Scholz, L. Nitschke and G. Henrion, Naturwissenschaften, 1989, 76, 71-72.

24 A. Chen, E. I. Rogers and R. G. Compton, Electroanalysis, 2009, 21, 29-35.

25 D. Khodagholy, V. F. Curto, K. J. Fraser, M. Gurfinkel, R. Byrne, D. Diamond, G. G. Malliaras, F. Benito-Lopez and R. M. Owens, J. Mater. Chem., 2012, 22, 4440-4443.

26 A. Kavanagh, R. Byrne, D. Diamond and K. J. Fraser, Membranes, 2012, 2, 16-39.

27 T. Torimoto, T. Tsuda, K.-i. Okazaki and S. Kuwabata, Adv. Mater., 2010, 22, 1196-1221.
28 S. Saricilar, D. Antiohos, K. Shu, P. G. Whitten, K. Wagner, C. Wang and G. G. Wallace, Electrochem. Commun., 2013, 32, 47-50.

29 A. F. Visentin and M. J. Panzer, ACS Appl. Mater. Interfaces, 2012, 4, 2836-2839.

30 M. Vuki, K.-K. Shiu, M. Galik, A. M. O'Mahony and J. Wang, Analyst, 2012, 137, 3265-3270.

31 K. Bratin, P. T. Kissinger, R. C. Briner and C. S. Bruntlett, Anal. Chim. Acta, 1981, 130, 295-311.

32 D. S. Silvester and R. G. Compton, Z. Phys. Chem., 2006, 220, 1247-1274. 